\title{
Necrotizing Soft Tissue Infection of the Foot: A Case Report
}

\author{
by Shelly A.M. Wipf, B.M. ${ }^{1}$, Robert M. Greenhagen, B.S. ${ }^{2}$, Denise M. Mandi, DPM ${ }^{3}$, Nicholas J. \\ Bevilacqua, DPM ${ }^{4}$, Lee C. Rogers, DPM ${ }^{5}$ 政
}

The Foot \& Ankle Journal 1 (4): 2

We report a rapidly spreading necrotizing foot infection in a healthy 37 year old male with associated sepsis and no identifiable portal of entry. Multiple debridements were required to control this infection which eventually necessitated a LisFranc's amputation. We review the current literature of the diagnosis and treatment of necrotizing soft tissue infections in the foot. Early diagnosis and appropriate treatment can preserve life and limb.

Key words: Necrotizing soft tissue infection, diabetic foot infection, LRINEC score

This is an Open Access article distributed under the terms of the Creative Commons Attribution License. It permits unrestricted use, distribution, and reproduction in any medium, provided the original work is properly cited. (The Foot \& Ankle Journal (www.faoj.org)

Necrotizing soft tissue infection or (NSTI) is a broad term that comprises a rare group of limb and life-threatening surgical emergencies. We report on an unusual case of a 37 year old, otherwise healthy male, who presented with a NSTI without a portal of entry. First described by Confederate army surgeon Joseph Jones in 1871 as "hospital gangrene," this condition is caused by a variety of organisms. These infections require operative debridement and appropriate antibiotic therapy, as well as fluid resuscitation and other supportive care.

\footnotetext{
Address correspondence to: Lee C. Rogers, DPM. Director, Amputation Prevention Center, Broadlawns Medical Center, 1801 Hickman Road, Des Moines, IA 50314.

Email: Lee.C.Rogers@gmail.com

1 Submitted while a $3^{\text {rd }}$ Year Podiatric Medical Student at Des Moines University - College of Podiatric Medicine and Surgery, 3200 Grand Avenue, Des Moines, IA 50312

2 Submitted while a $4^{\text {th }}$ Year Podiatric Medical Student at Des

Moines University - College of Podiatric Medicine and Surgery, 3200

Grand Avenue, Des Moines, IA 50312

3 Chairman; Department of Surgery, Broadlawns Medical Center, 1801 Hickman Road, Des Moines, IA 50314

4 Amputation Prevention Center at Broadlawns Medical Center, 1801

Hickman Road, Des Moines, IA 50314

5 Director; Amputation Prevention Center at Broadlawns Medical

Center, 1801 Hickman Road, Des Moines, IA 50314
}

The term "necrotizing fasciitis" was coined by Wilson in 1952. ${ }^{2}$ Today, most authors prefer the term "necrotizing soft tissue infection," or NSTI.

\section{Case Report}

A 37-year-old white male presented to the Emergency Department complaining of a very painful and swollen right foot. The patient noted that the swelling and pain had been increasing to the lower leg and up to the knee. There had been increasing redness and some clear drainage from the right foot. The patient had been evaluated at an urgent care clinic five days earlier with a complaint of severe pain to the arch. At that time, there was no evidence of an infectious process and the patient was diagnosed with plantar fasciitis. He was given a non-steroidal, anti-inflammatory and a narcotic for pain. 


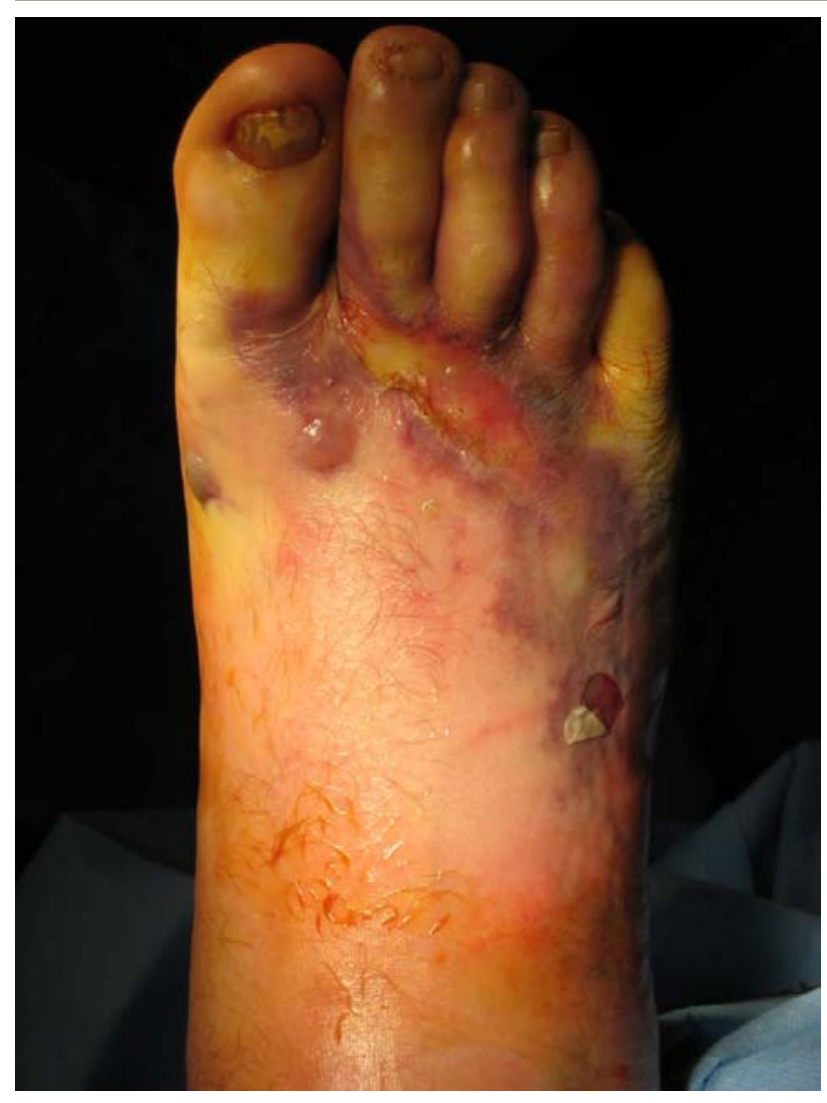

Figure 1 The initial presentation of the foot. There is extensive cyanotic skin changes and bullae formation.

The patient denied a history of trauma or laceration to the foot, as well as any illicit drug use. The patient had a negative history of recent respiratory or genitourinary infections, although he had noticeably poor dentition. His past medical history was unremarkable. The patient reported allergy to penicillin. Significant vital signs included a temperature of $38.1^{\circ} \mathrm{C} / 100.6^{\circ} \mathrm{F}$, and tachycardia at a rate of 124 . Examination of the right lower extremity revealed non-palpable pedal pulses secondary to edema, although capillary refill was normal. No lacerations, punctures, or abrasions were observed. There were hemorrhagic blisters on the dorsal and plantar forefoot that exuded no purulence. (Fig 1)

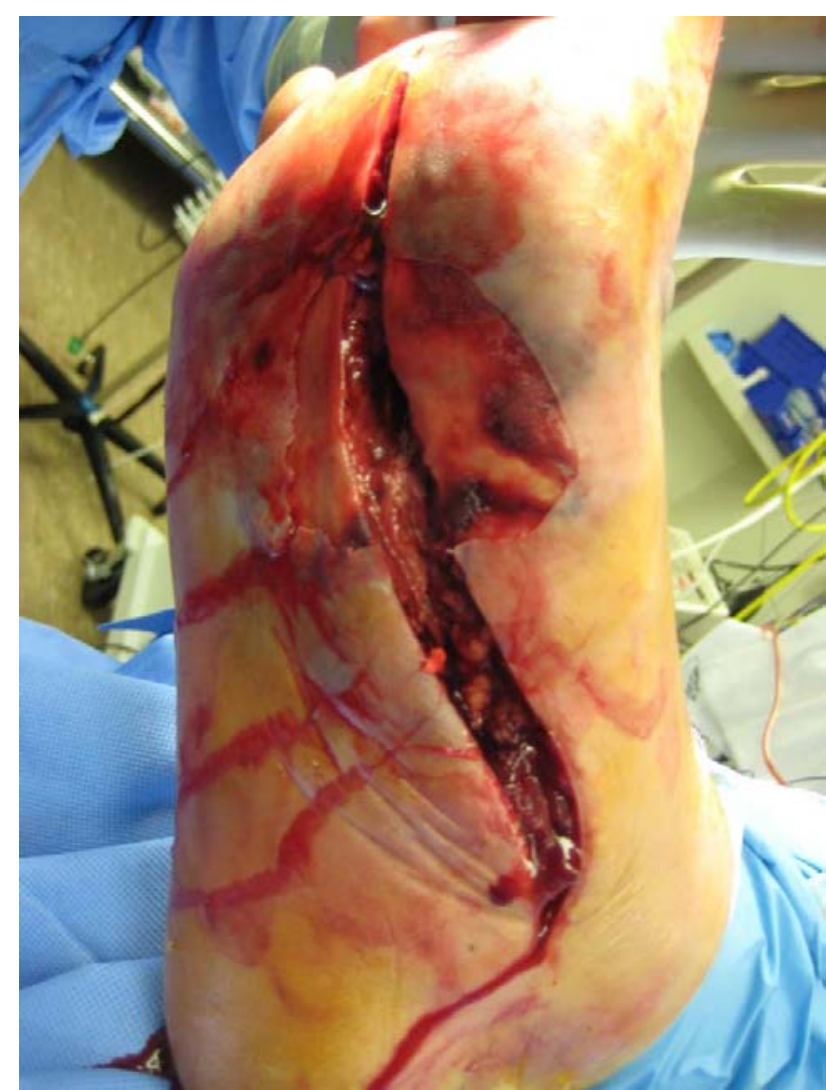

Figure 2 Intra-operative photo of the plantar incision and drainage showing extensive myonecrosis with minimal bleeding.

Ecchymosis was observed at the medial plantar arch and the lateral sub-fibular region. Radiographs failed to reveal any bony pathology or subcutaneous gas. Admission laboratory studies were performed. Results included a white blood cell count of 14,700 with $18 \%$ bands, hemoglobin and hematocrit of 13.8 and 39.3 respectively, sodium of 131 , potassium of 3.3 , and blood glucose of 197. C-reactive protein was markedly elevated at 23.6. Blood cultures were drawn and Gram stain revealed Gram positive cocci in chains (later identified as Streptococcus Group A).

The patient was taken to the operating room for incision and drainage, at which time seropurulence was expressed from all major compartments of the foot. (Fig 2) 


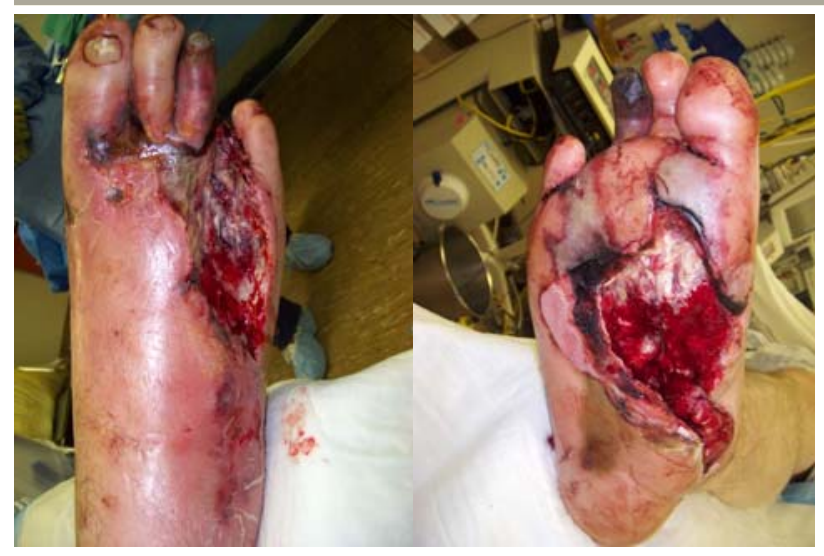

Figures 3,4 Appearance of the foot after amputation of the fourth toe prior to the fourth surgical debridement. There is continued necrosis of soft tissue and loss of tissue viability to toes 1-3.

Lack of resistance of fascial planes to blunt dissection was noted. Multiple coagulated veins were present in the subcutaneous tissue. No bleeding occurred. Following hydroscapel debridement and irrigation with pulse lavage, the wound was packed open with Iodoform gauze. The patient was placed on Linezolid, Clindamycin, and Aztreonam.

On hospital day three, it was discovered that the cultures from the foot revealed Group A Streptococcus. The antibiotic spectrum was narrowed to Clindamycin. Over the next six days the patient underwent two subsequent debridements involving the removal of digits 2,3 , and 4 which had become necrotic. (Figs. 3,4) Negative pressure wound therapy was employed after the amputations. On hospital day nine, the infection was controlled and no further necrosis was observed. Attention was focused on limb salvage and creation of a functional foot. On day sixteen, the patient underwent a LisFranc disarticulation amputation with primary closure utilizing local tissue transfer. (Figs. 5,6)

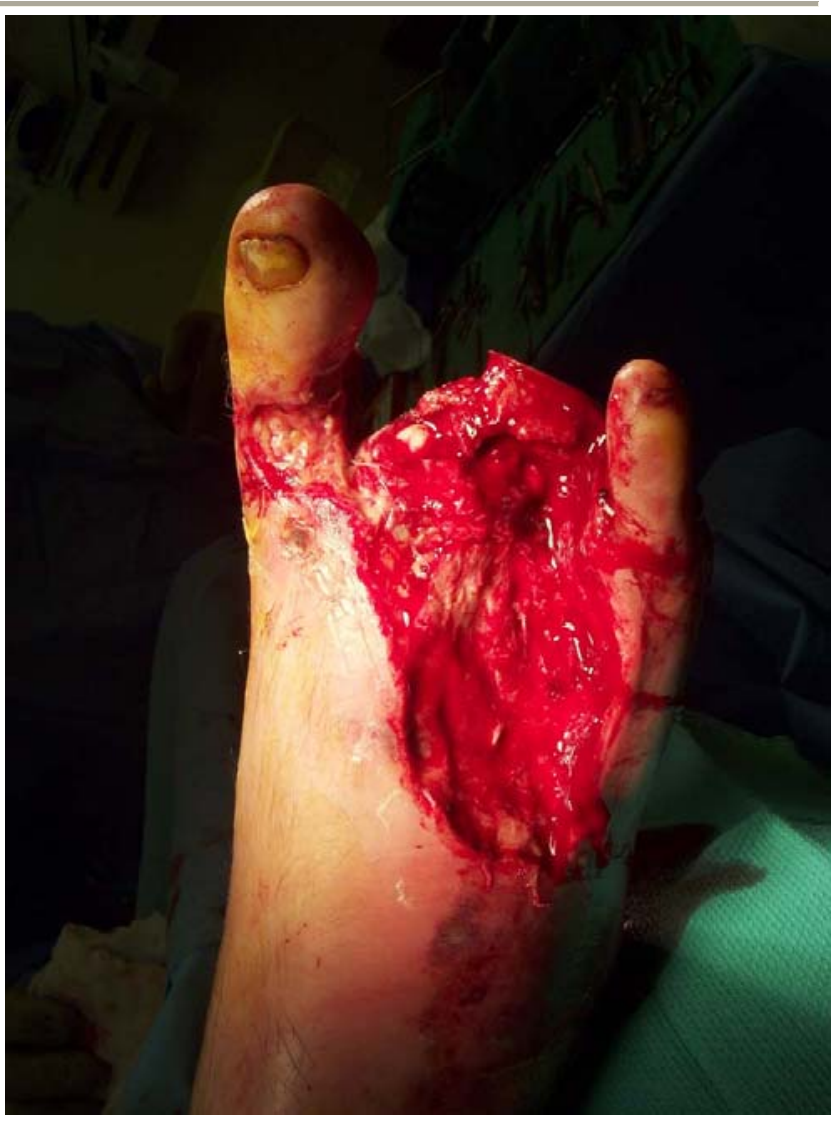

Figure 5 After four surgical debridements with amputation of toes 2,3 , and 4 , there is now viable bleeding tissue that is free from infection and necrosis.

\section{Discussion}

NSTIs are characterized by bacterial invasion leading to thrombosis and suppuration of the subcutaneous vasculature. The angiothrombosis effectively decreases blood supply, causing liquefactive necrosis of the subcutaneous tissue, fascia, and eventually the musculature. The angiothrombosis renders the delivery of parenteral antibiotic therapy ineffective, necessitating emergent operative debridement of gangrenous fascia, fat, and blood vessels to the extent of healthy, bleeding and viable tissue. 


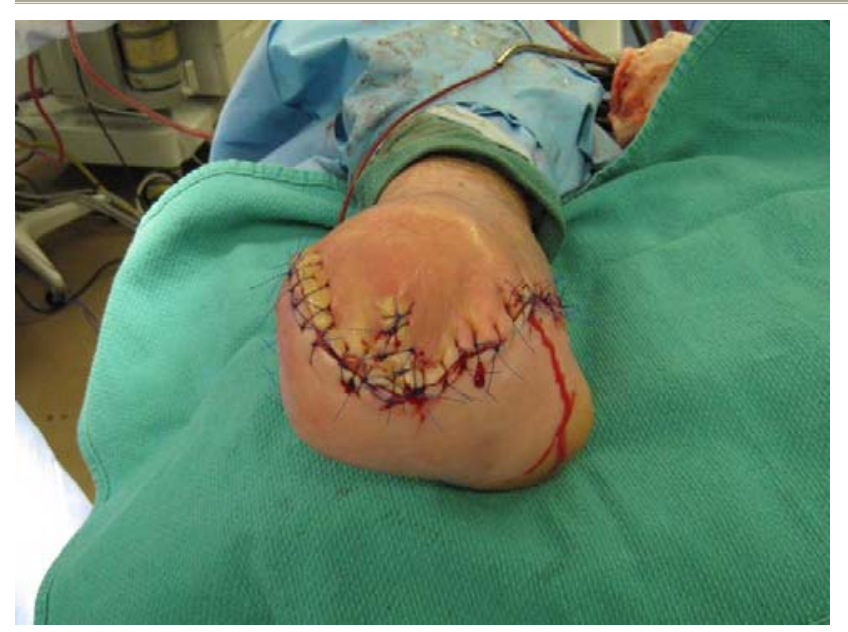

Figure 6 The forefoot was disarticulated at the LisFranc joint and the medial and lateral forefoot skin was used as a fasciocutanous flap to cover the defect.

Debridement reveals gray necrotic fascia, lack of bleeding during dissection, muscle fascia that gives way to blunt dissection, and foul-smelling "dish-water" purulence. ${ }^{3}$ The clinical course of NSTI is often not as rapid as would be expected from the media reports of "flesh-eating bacteria." Although fever and tachycardia may begin at 24 hours, the pathognomonic features of skin sloughing, bullae, and cutaneous gangrene do not usually occur until the fourth or fifth day. ${ }^{4,5,6}$ Our patient demonstrated this course of disease.

The bacteriologic isolates of necrotizing soft tissue infection have evolved since early descriptions. Giuliano and colleagues ${ }^{7}$ classified NSTI into two groups: Type I is a polymicrobial infection consisting of anaerobic bacteria and facultative anaerobic bacteria; and Type II is a monomicrobial infection of Group A Streptococcus. However, in their original report Giuliano, et al, describe one Type II infection with Group A Streptococcus in combination with Staphylococcus aureus. Recent reports have isolated non-Group A Streptococcus in monomicrobial infections, including Staphylococcus aureus, Clostridium, Pseudomonas aeruginosa, Escherichia coli, and Serratia marscesans. ${ }^{8,9}$
The exact microbiological etiology of NSTI is a moot point, however, when the patient presents in the fulminant phase of disease. At that most critical period only two types of NSTI must be differentiated: pure clostridial myonecrosis and non-clostridial infection. ${ }^{11}$ Clostridial infection invades muscle tissue and carries a four-fold risk of mortality and limb loss. ${ }^{22}$ NTSI are associated with several co-morbidities. The most common factors predisposing patients to NSTI are diabetes mellitus, peripheral vascular disease, obesity, alcohol abuse/hepatic disease, malignancy, immunosuppressant therapy, and intravenous drug abuse. ${ }^{3,8,10,11}$ Portals of entry for NTSI include sites of pre-existing ulcerations, recent surgical wounds, traumatic wounds, sites of subcutaneous insulin injection or illicit drug injection, and minor abrasions, burns, lacerations, or insect bites. ${ }^{8,11}$ In urban hospital settings, the incidence of NSTI secondary to intravenous injection of illicit drugs has been increasing in recent years, necessitating the need to question the patient's social history. The physician must be aware of the practice of "skin popping," in which a substance such as heroin is injected directly into the subcutaneous tissues. Infections associated with intravenous drug abuse are caused by skin flora and anaerobic bacteria. Oropharyngeal bacteria such as Streptococcus viridans and Eikenella corrodens can also cause NSTI. This is due to the practice of abusers licking their needles to avoid wasting any drug. ${ }^{12}$ Rapid differentiation between NSTI and cellulitis or abscess is paramount in reducing morbidity and mortality. The most common presenting signs of NSTI are nonspecific signs of inflammation such as erythema, edema, and pain. Wong, et al, ${ }^{3}$ describe a "triad" of exquisite pain (97.8\% of patients), swelling (92.1\%), and fever (79.8\%). In their matched consecutive case series of 21 NSTI cases and 21 non-NSTI controls, Wall and colleagues ${ }^{13}$ identified tense edema (38\% vs $0 \%, \mathrm{p}=0.003)$ and bullae $(24 \%$ vs $0 \%$; $\mathrm{p}=0.049)$ as the most specific physical findings. Present in four-fifths of patients with document NSTI, the three most specific "hard" clinical signs of NSTI are bullae formation, skin necrosis, and crepitance. ${ }^{11}$ 
Some patients do not possess any of these signs at presentation. Imaging, such as plain radiography ${ }^{20}$ ultrasound, ${ }^{14,15} \mathrm{CT}$ scans, ${ }^{16}$ and $\mathrm{MRI}^{17,18}$ show nonspecific changes and are often not significant until late in the course of the disease. Near-infrared spectroscopy is a newer, less available modality that can diagnose NSTI in the lower limbs by measuring tissue hypoxia secondary to microvascular thrombosis and necrosis. At a tissue oxygen saturation reading of less than $70 \%$, the test is $100 \%$ sensitive and $97 \%$ specific for NSTI. ${ }^{19}$

McHenry reported the cumulative mortality of NSTI to be $34 \% .^{8}$ Death can result from disseminated intravascular coagulation, acute respiratory distress syndrome, or multiple organ failure in response to overwhelming sepsis. ${ }^{20}$ Anaya, et al, ${ }^{21}$ found the most significant predictors of mortality to be white blood cell count greater than $30,000 \times 10^{3} / \mu \mathrm{L}$ and creatinine greater than $2 \mathrm{mg} / \mathrm{dL}$. The single most important determinant of survival and limb preservation in NSTI is the amount of time elapsed between initial presentation and surgical debridement. McHenry reported the average time from admission to operation in non-survivors to be 90 hours, while in survivors the elapsed time was only 25 hours $(p=0.0002) .{ }^{8}$ In a retrospective review of 68 patients, Bilton, et $\mathrm{al}^{22}$ reported $4.2 \%$ mortality for patients undergoing early surgical debridement and $38.0 \%$ mortality for those with delayed treatment $(\mathrm{p}=0.0007)$.

Wong and colleagues ${ }^{23}$ developed the Laboratory Risk Indicator for Necrotizing Fasciitis (LRINEC) scoring system for accurately identifying NSTI based on lab values at time of hospital admission (Table 1).

This scoring system was derived using univariate and multivariate logistic regression analysis of 89 patients with surgically confirmed NSTI and 225 control patients with cellulitis or subcutaneous abscess. The LRINEC score considers the lab values for C-reactive protein, total white cell count, hemoglobin, sodium, serum creatinine, and glucose.

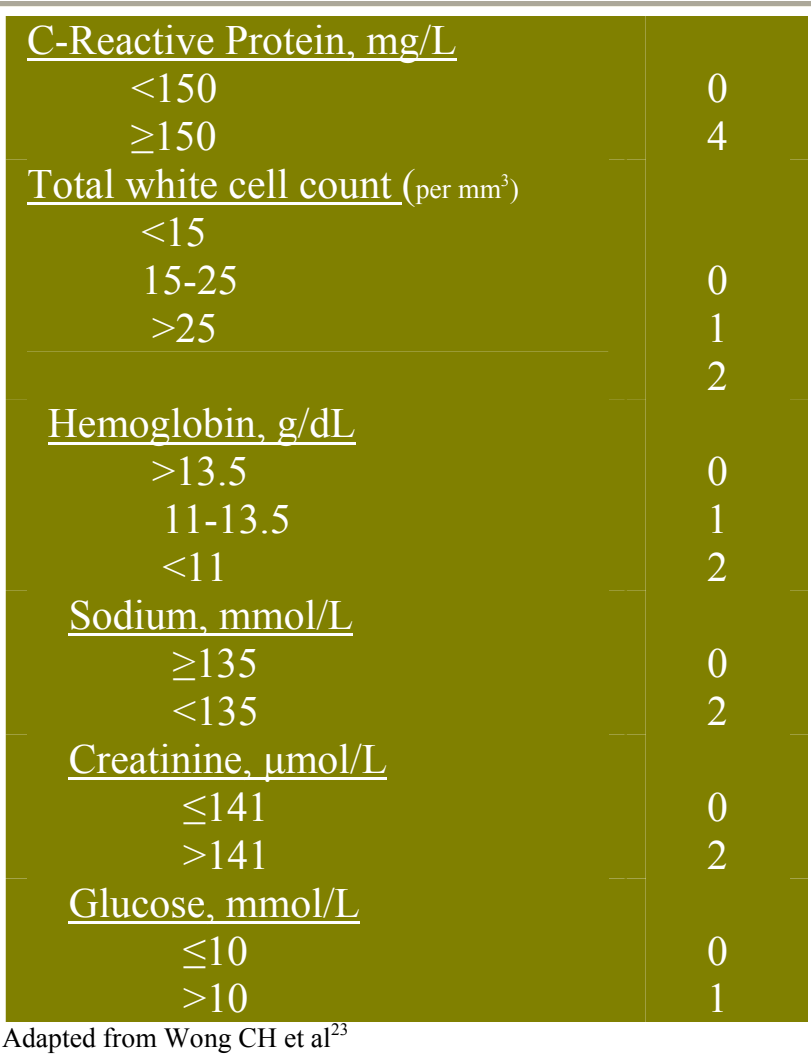

Table 1: Laboratory Risk Indicator for Necrotizing Fasciitis (LRINEC) Score

For patients whose score was greater than 6 points (range 0-13), the LRINEC score had a positive predictive value of $92.0 \%$ and a negative predictive value of $96.0 \%$. Patients were stratified into risk categories: low risk for a score of $\leq 5$, moderate risk for a score of 6-7, and high risk for a score $\geq 8$. The authors' suggested algorithm for patients in the low risk category is intravenous antibiotics and continued monitoring of $\mathrm{CBC}$ and CRP. For intermediate risk patients, they suggest urgent MRI, frozen section biopsy, or "finger test," a bedside procedure in which a 2-cm incision is made down to deep fascia and the wound inspected for lack of bleeding, presence of "dishwater pus," and lack of resistance to blunt dissection. For patients at high risk with a score of 8 or higher, the recommendation is emergent operative debridement. Our patient presented to the Emergency Department with a LRINEC score of 7 , placing him in the moderate risk category. 


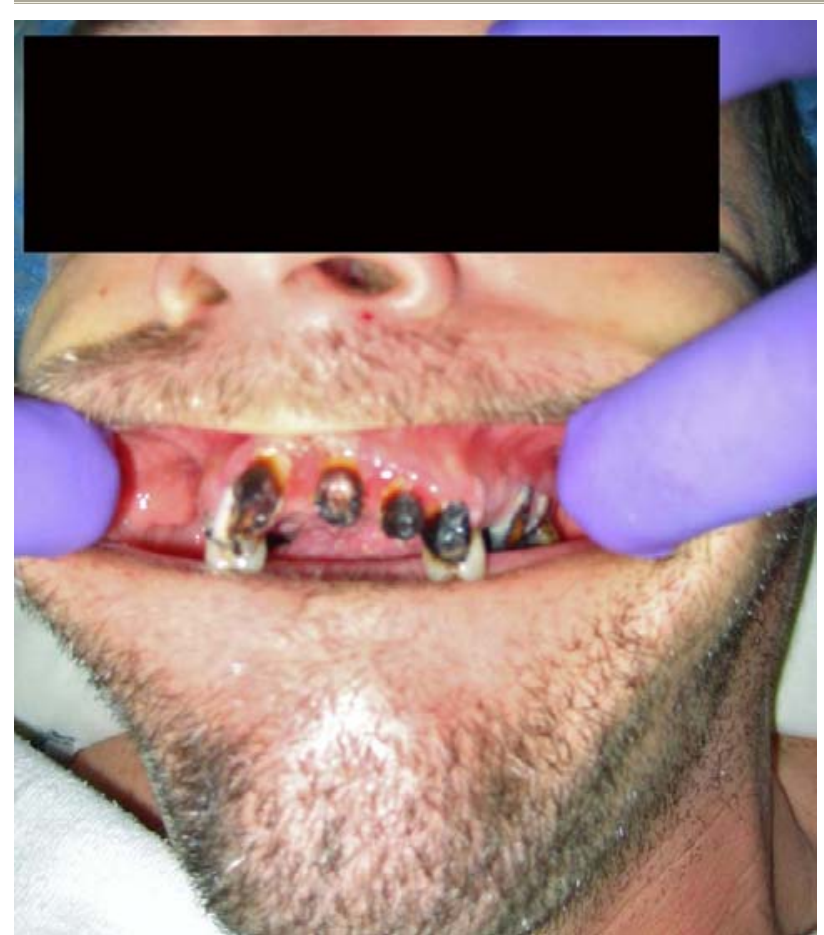

Figure 7 A view of the patient's oropharyngeal cavity exhibiting severe tooth decay and ginigivitis.

The mainstay of treatment for necrotizing soft tissue infections is rapid surgical debridement followed by appropriate antibiotic therapy after initial resuscitation with fluid and electrolyte replacement and/or blood transfusion. Often multiple debridements are necessary. In the report by Wong, et $\mathrm{al}^{23}$ survivors underwent a mean of 2.7 debridements (range, 0-9) to control the infective process. While amputation was not found to reduce mortality, patients who underwent amputation had to undergo fewer operations to control the infection and to achieve wound coverage (2.59 compared with 3.45; $\mathrm{p}<0.05) .{ }^{3}$ Before cultures are available, empiric antibiotic therapy that is effective against gram positive, gram negative, and anaerobic bacteria should be initiated early. McHenry and colleagues $^{8}$ suggest using single antibiotics such as imipenem-cilastin, ticarcillin-clavulanate, ampicillin-sulbactam, or piperacillin-tazobactam.
In their series, Bosshardt, et al, ${ }^{12}$ employed triple antibiotic therapy consisting of ampicillin or high dose penicillin $G$, an aminoglycoside, and clindamycin or metronidazole.

They suggested the combination of ampicillinsulbactam and high dose penicillin $G$ for drug abusers due to the higher percentage of infections caused by oropharyngeal flora and Clostridial species.

The use of hyperbaric oxygen to treat NSTI may be beneficial. Wilkinson, et al, ${ }^{24}$ reported a study of 44 patients with NSTI. Thirty-three patients received $\mathrm{HBO}_{2}$ in addition to standard care and eleven patients received only standard care. They found a significant reduction in the incidence of amputation $(p=0.05)$ and an $83 \%$ reduction in the relative risk of death for the $\mathrm{HBO}_{2}$ group, which corresponds to a number needed to treat (NNT) of 3 .

The diagnosis of necrotizing soft tissue infection can be elusive and requires an astute clinician to recognize it in its early stages. This will help to increase the patient's chance of survival and limb preservation. The clinician must realize that the pathognomonic skin changes do not appear until four or five days after initial presentation of pain, swelling and erythema. Though unconfirmed, we speculate that our case of NTSI was initiated by bacteremia from poor dentition. (Fig 7).

In our case, rapid identification of the NSTI and septicemia contributed to our ability to preserve life and limb, through a foot sparing amputation. The patient underwent a dental consultation immediately following surgery to evaluate necrotizing gingivitis and severe tooth decay. 


\section{References}

1. Jones J. Investigation upon the nature, causes and treatment of hospital gangrene as it prevailed in the Confederate armies, 1861-1965. In: Surgical Memories of the War of Rebellion New York: United States Sanitary Commission, 1871.

2. Wilson B: Necrotizing fasciitis. Am Surg. 18 :416-431, 1952.

3. Wong $\mathrm{CH}$ et al: Necrotizing Fasciitis: Clinical

Presentation, Microbiology, and Determinants of Mortality. JBJS. 85-A(8): 1454-1460, 2003.

4. Beathard GA: Necrotizing Fasciitis Due to Group A $\beta$ hemolytic Streptococci. Arch Intern Med. 120:63-67, 1967. 5. Janevicius RV et al: Necrotizing fasciitis. Surg Gynecol Obstet. 154:97-102, 1982.

6. Koehn GC: Necrotizing fasciitis. Arch Dermatol. 114:581-583, 1978.

7. Giuliano, A et al: Bacteriology of necrotizing fasciitis. Am. J Surg. 134: 52-56, 1977.

8. McHenry CR et al: Determinants of Mortality for Necrotizing Soft-Tissue Infections. Annals of Surgery. 221(5): 558-565, 1995.

9. Singh $G$ et al: Bacteriology of Necrotizing Infections of Soft Tissues. Aust N.Z. J. Surg. 66: 747-750, 1996.

10. Wang KC et al: Necrotizing Fasciitis of the Extremities. J Trauma. 32: 179-182, 1992.

11. Elliott D et al: Necrotizing Soft Tissue Infections: Risk factors for mortality and strategies for management. Ann Surg. 224: 672-683, 1996.

12. Bosshardt TL et al: Necrotizing soft-tissue infections. Arch Surg. 131: 846-854, 1996.

13. Wall DB; et al: Objective criteria may assist in distinguishing necrotizing fasciitis from nonnecrotizing soft tissue infection. Am J Surg. 179: 17-21, 2000.
14. Tsai CC et al: Early diagnosis of necrotizing fasciitis by utilization of ultrasonography. Kaohsiung J Med Sci. 12:235-240, 1996.

15. Yen ZS et al: Ultrasonographic screening of clinicallysuspected necrotizing fasciitis. Acad Emerg Med. 9(12): 1448-1451, 2002.

16. Wysoki MG et al: Necrotizing Fasciitis: CT

Characteristics. Radiology. 203: 859-863, 1997.

17. Brothers TE et al: Magnetic resonance imaging differentiates between necrotizing and non-necrotizing fasciitis of the lower extremity. J Am Coll Surg. 187: 416421, 1998.

18. Schmid MR et al: Differentiation of necrotizing fasciitis and cellulitis using MR imaging. Am J Roentgenol. 70: 615620, 1998

19. Wang TL et al: Role of Tissue Oxygen Saturation Monitoring in Diagnosing Necrotizing Fasciitis of the Lower Limbs. Ann Emerg Med. 44: 222-228, 2004. 20. Fontes RA et al: Necrotizing Soft Tissue Infections. J Am Acad Orthop Surg. 8(3): 151-158, 2000.

21. Anaya DA et al: Predictors of Mortality and Limb Loss in Necrotizing Soft Tissue Infections. Arch Surg. 140: 151157, 2005.

22. Bilton $\mathrm{BD}$ et al: Aggressive surgical management of necrotizing fasciitis serves to decrease mortality: a retrospective study. Am Surg. 64(5): 397-401, 1998. 23. Wong $\mathrm{CH}$ et al: The LRINEC( Laboratory Risk Indicator for Necrotizing Fasciitis) score: A tool for distinguishing necrotizing fasciitis from other soft tissue infections. Crit Care Med. 32(7): 1535-1541, 2004. 24. Wilkinson D et al: Hyperbaric Oxygen Treatment and Survival From Necrotizing Soft Tissue Infection. Arch Surg. 139: 1339-1345, 2004. 